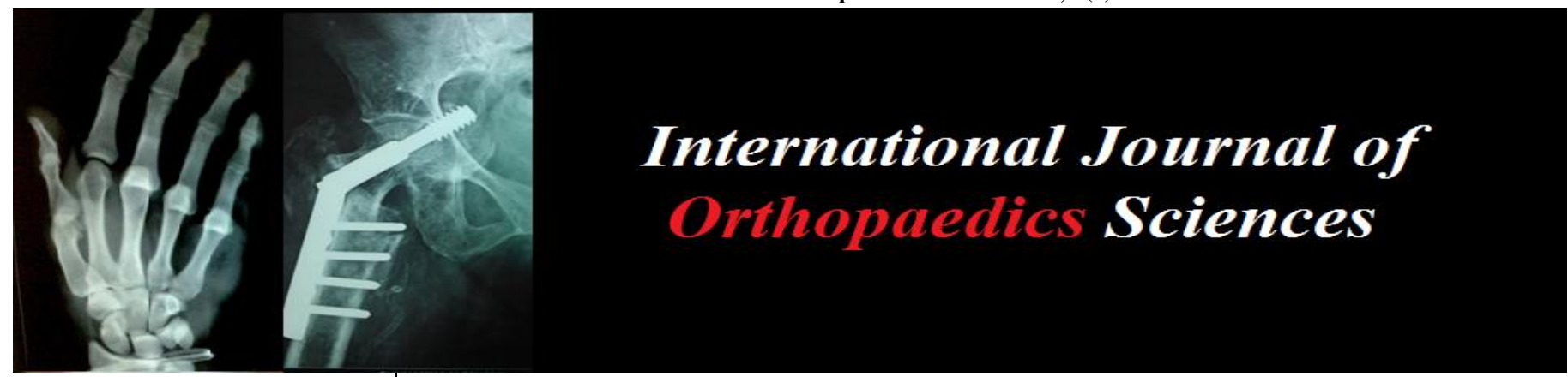

ISSN: $2395-1958$

IJOS 2019; 5(3): 435-440

(C) 2019 IJOS

www.orthopaper.com

Received: 05-05-2019

Accepted: 10-06-2019

Dr. Vadhiraj Krishna JB

Junior Resident, Department of Orthopedics, Mallya Hospital,

Bengaluru, Karnataka, India

Dr. M Rama Subba Reddy Senior Consultant, Department of Orthopedics, Mallya Hospital Bengaluru, Karnataka, India

Dr. Rohan Natthuji Kumre Senior Resident, Department of Orthopedics, Mallya Hospital, Bengaluru, Karnataka, India
Correspondence

Dr. Vadhiraj Krishna JB

Junior Resident, Department of

Orthopedics, Mallya Hospital,

Bengaluru, Karnataka, India

\section{Surgical outcome of Schatzker type 5 and type 6 tibial plateau fractures treated by plating with or without Cannulated Cancellous screws augmentation: A prospective study of $\mathbf{4 5}$ cases}

\author{
Dr. Vadhiraj Krishna JB, Dr. M Rama Subba Reddy and Dr. Rohan \\ Natthuji Kumre
}

DOI: https://doi.org/10.22271/ortho.2019.v5.i3h.1568

\section{Abstract}

Background: Knee joint is an important joint as it is involved in varied functions like load bearing, walking, running, sitting etc. Tibial plateau fractures are encountered in high velocity trauma, which are associated with high morbidity and mortality. Complications include fat embolism, compartment syndrome, ARDS, which necessitates early fixation. Among tibial plateau fractures, Schatzker type V and type VI are complex injuries and treated by internal fixation by plates and screws with or without augmentation by cannulated cancellous screws.

Aim: To analyze the post-operative surgical outcomes of Schatzker type V and type VI tibial plateau fractures treated by open reduction and internal fixation by plating with or without augmentation by cannulated cancellous screws.

Materials and Methods: Patients were admitted in Mallya Hospital, Bangalore in between October 2016 to November 2018. Total of 45 Patients with Schatzker type V and type VI tibial plateau fractures included in the study. Closed fractures and open type I and type II without severe soft tissue injury were included. The patients were recruited to the study after due consent. Patients were operated by open reduction and internal fixation by plating (Dual LCP, Single LCP, Single LCP + CC SCREWS) through anterolateral approach for lateral plate and posteromedial approach for medial plate. Further patients were followed up postoperatively, 6 weeks, 3 months and 6 months. Surgical outcomes were analyzed as subjective, clinical, radiological and functional outcomes based on Honkonen and Jarvinen Criteria.

Results: 45 cases were included in study, out of which 7 were lost to follow up. Minimum age of the patient was 22 years and maximum 67 years. There were 29 males and 16 female patients. 24 patients were Schatzker type V and 14 patients were Schatzker type VI. 18 patients were operated with single plating, 12 were by single plating $+\mathrm{CC}$ screw, 8 were operated with dual plating.

There was no statistical difference in the subjective ( $\mathrm{p}$ value- 0.345$)$ functional. (P value- 0.221 ),

Clinical ( $\mathrm{p}$ value-0.153), radiological ( $\mathrm{p}$ value-0.590) outcomes between type $\mathrm{V}$ and type VI fractures.

Conclusion: We concluded that Open reduction and internal fixation by plating (dual LCP, single LCP with or without cancellous cannulated screws) of closed and open (type I and type II) Schatzker type V and VI tibial plateau fractures is an effective method of treatment.

Prognosis of this complex fracture depends on

- The degree of articular reconstruction

- The integrity of the soft tissue envelope

- $\quad$ Post-operative physiotherapy

We found no statistical difference in assessing the Schatzker type V and VI fracture patterns. Comparable Results were achieved in radiological, clinical, subjective and functional outcomes based on Honkonen and Jarvinen criteria.

Keywords: Schatzker classification, tibial plateau, honkonen and jarvinen criteria

\section{Introduction}

The luxuries of our modern-day life have come at the cost of rapidly increasing industrialization, urbanization and mechanization - so also the traumatic cases are on the rise. Fractures of tibial plateau involve a major weight bearing joint, the knee joint.

$$
\sim 435 \sim
$$


These are serious injuries that frequently result in functional impairment, as they affect knee alignment, stability and movement. Tibial plateau fractures make about $1 \%$ of all fractures and $8 \%$ of the fractures in elderly. Most injuries affect lateral tibial condyle (55 to $70 \%$ ) and isolated medial condyle fractures occur in 10 to $23 \%$ whereas the involvement of bicondylar lesions is found in 10 to $30 \%$ of the reported series ${ }^{[1]}$.

\section{Main challenges encountered in the treatment of tibial plateau fractures are}

- These high energy fractures are associated with extensively damaged soft tissue envelope leading to increased incidence of compound injuries which results in increased complications following open reduction and internal fixation. Also, comminution of metaphysis and articular surface makes anatomical reduction difficult. The resulting in-congruency of articular surface leads to early secondary osteoarthritis.

- In metaphysis, fixation is less satisfactory which may result in early loosening of implant.

- Comminuted fractures create difficulty in achieving rigid fixation due to poor purchase and are less optimal to permit weight bearing or even early joint mobilization.

In the past two decades, with improvements in surgical techniques and implants, there has been an ever-increasing trend towards surgical management of these injuries. With no proven uniform successful method of treatment, Nevertheless, tibial plateau fractures remain challenging because of their number, variety and difficulty in fixation. Despite a plethora of articles, written in the past 50 years that have addressed the problems of classification and results of various treatments, the optimal method of management remains controversial ${ }^{[2]}$.

Numerous investigators report satisfactory results using either closed or open treatment methods, especially for the less severe type injuries that occur as a result of low energy trauma (Schatzker types 1-4). For the more severe form of injury which results in bicondylar tibial plateau fractures (Schatzker type V and VI) the ideal mode of treatment is always debatable. Conservative mode of management, Open reduction and internal fixation, closed reduction and percutaneous fixation, Hybrid external fixation have all been suggested [3]. Age, skin condition, soft tissue status, osteoporotic bones further complicate the healing process.

\section{Before deciding the line of treatment, one must bear in mind the following facts}

1. Extent of damage in tibial plateau fracture is often greater than what is seen on $x$-ray.

2. Malunion is extremely common whereas non-union is unknown.

3. Painful knee and stiffness are the most serious and common complications of the tibial plateau fracture.

In this study, we intend to outline the various principles of surgical management of tibial plateau fracture type 5 and type 6 by internal fixation by plates and screws with or without augmentation by cannulated cancellous screws and to put forward fresh clinical material to evaluate the results of surgical management.

\section{Materials and Methods}

- The study was conducted in the department of orthopedics, Mallya Hospital, Bengaluru. This study consisted of 45 Patients visiting Outpatient and emergency department of Mallya hospital. Patients diagnosed with Schatzker type 5 and type 6 tibial plateau fractures who were operated during the period from October 2016 to November 2018 were included in the study. Follow up ranged from 6 to 18 months. All fractures were post traumatic, between 18-70 years of age group, having isolated closed/open fracture (Gustilo Anderson type 1 and 2). the patients excluded were those having any other ipsilateral lower limb fracture, Patients with severe head injury, Gustilo Anderson type 3 open fracture, Patients with permanent neurovascular deficits and Pathological fractures.

\section{Surgical procedures}

With the patient in supine position under anaesthesia, tourniquet was applied, Reduction of fracture was done; typically, medial column fixed first. If medial condyle was severely comminuted, then lateral column was fixed first to maintain length.

Lateral condyle was exposed, through an anterolateral approach, with meticulous Soft tissue handling. Correction of articular comminution or depression and provisional stabilization of the fracture was done. Large periarticular clamps were used to effect compression between the medial and lateral condyles. Definitive anterolateral fixation with a precontoured proximal tibial locking compression plate with or without cannulated cancellous screws augmentation, whose proximal fixation allows capture of the medial condylar segment.

The minimally invasive posteromedial approach was used and a 1 inch long longitudinal skin incision was made, medial or posteromedial fragment exposed sub periosteally by elevating pes anserinus with periosteal elevator. Medial fragment was fixed with a precontoured proximal tibial locking compression plate.

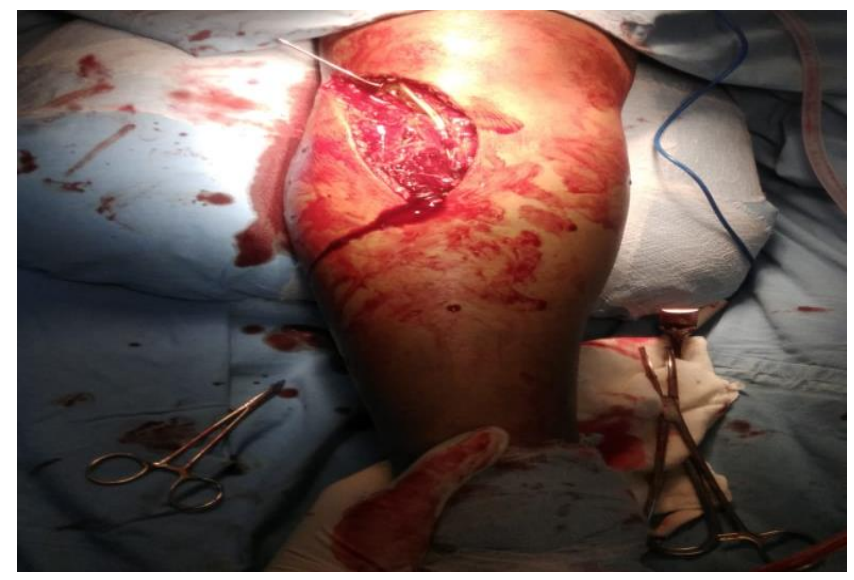

Fig 1: Lateral Plate Placement 


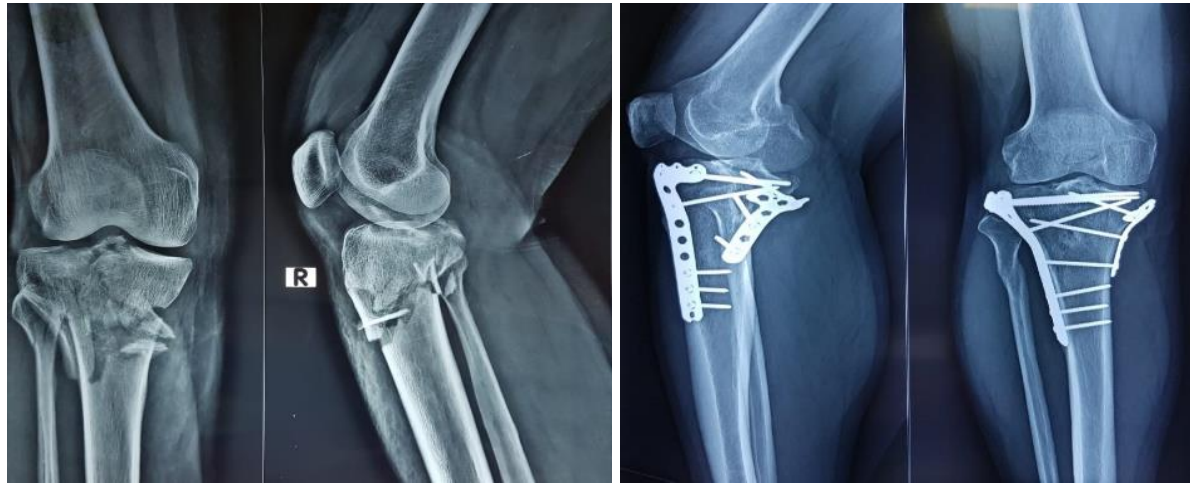

Fig 2: Pre op and follow up x-rays
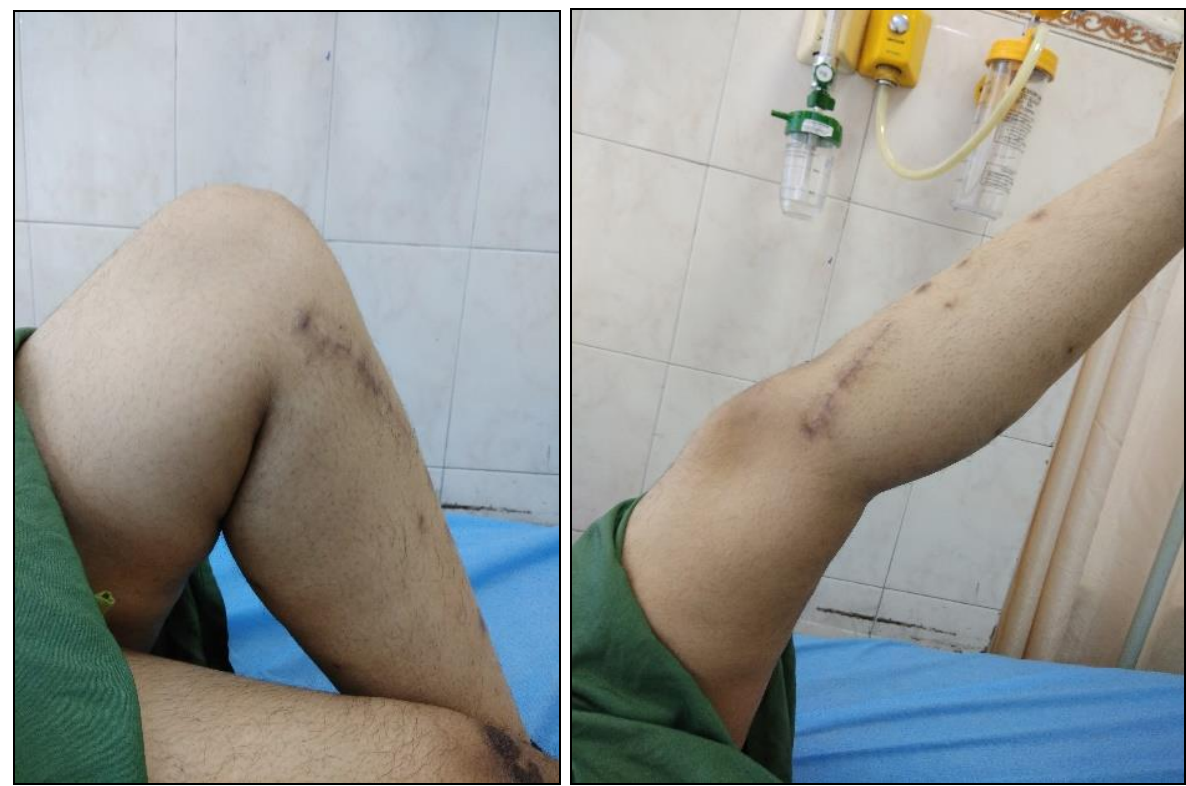

Fig 3: Knee range of movements

\section{Post-operative protocol}

Post operatively, patients were started with early knee range of movement exercises and quadriceps strengthening exercises ( $3^{\text {rd }}$ day) depending on pain tolerance of patient. Toe touching walking with crutch and walker support for 8 weeks followed by partial and full weight bearing walking depending upon radiological healing of fracture.

\section{Results}

In our clinical study, there were 16 females (35.6\%) and 29 males $(64.4 \%)$, ratio of male to female being 1.8:1. Minimum age was $21 y r s$ and maximum age was $67 y$ rs. mean age of our study was $46.06+13 y r s .7$ patients were lost in the follow up.

RTA was most common mode of injury, 41 (91.1\%) of the fractures occurred as a result of high energy road traffic accidents, $4(8.9 \%)$ due to fall from height. Right side \& left side involvement was $24(53.3 \%)$ and $21(46.7 \%)$ respectively. majority of the patients $36(80 \%)$ had closed fractures, $4(8.9 \%)$ had open fracture type I and $5(11.1 \%)$ patients had open type II fractures.29 (64.4\%) patients had Schatzker type $\mathrm{V}$ fracture and $16(35.6 \%)$ patients had Schatzker type VI fracture average knee moment (flexion) was $119^{\circ}$, ranging from a minimum of 80 degrees to 140 degrees. subjective results $31.6 \%$ had excellent, $31.6 \%$ had good, $26.3 \%$ had fair and $10.5 \%$ had poor results, most of the patients were symptom free at the end of follow up.

Clinical outcome was $42.1 \%$ had excellent, $34.2 \%$ had good, $13.2 \%$ had fair and $10.5 \%$ had poor results. Only 2 patients had extension lag of more than 10 degrees by the end of 6 months follow up. radiological outcome was $44.7 \%$ had excellent, $31.6 \%$ had good, $13.2 \%$ had fair and $10.5 \%$ had poor results. Articular step of more than $5 \mathrm{~mm}$ were noted in 3 patients who had poor outcomes. The same 3 patients also showed $>50 \%$ decrease in joint space compared to opposite knee and varus/valgus tilt $>10$ degree. 2 patients showed condylar widening of $>10 \mathrm{~mm}$.

Functional results were $50 \%$ had excellent, $13.2 \%$ had good, $21.1 \%$ had fair and $15.8 \%$ had poor results. Only 2 patients in whom anatomic reduction was not achieved were unable to climb stair and squat. Walking was normal in most of them with slight limp in 6 patients. 3 patients needed the assistance of a stick. All patients returned to their preoperative employment successfully of 38 patients available for follow up 18 patients were operated with single plating, 12 were operated with single plating $+\mathrm{CC}$ screw, 8 were operated with dual plating.

The functional outcomes were evaluated, there were no statistical difference in the functional outcome between the different procedures. ( $\mathrm{p}$ value-0354). There was no statistical difference in the subjective ( $\mathrm{p}$ value-0.345) functional. ( $\mathrm{p}$ value-0.221), clinical ( $\mathrm{p}$ value-0.153), radiological ( $\mathrm{p}$ value0.590 ) outcome between type V and type VI, Majority of the patients $91.1 \%$ did not develop any complication, $4.4 \%$ of the patients developed infection, $2.2 \%$ of the patients had Impending Compartment syndrome and $2.2 \%$ of the patients had wound dehiscence. 


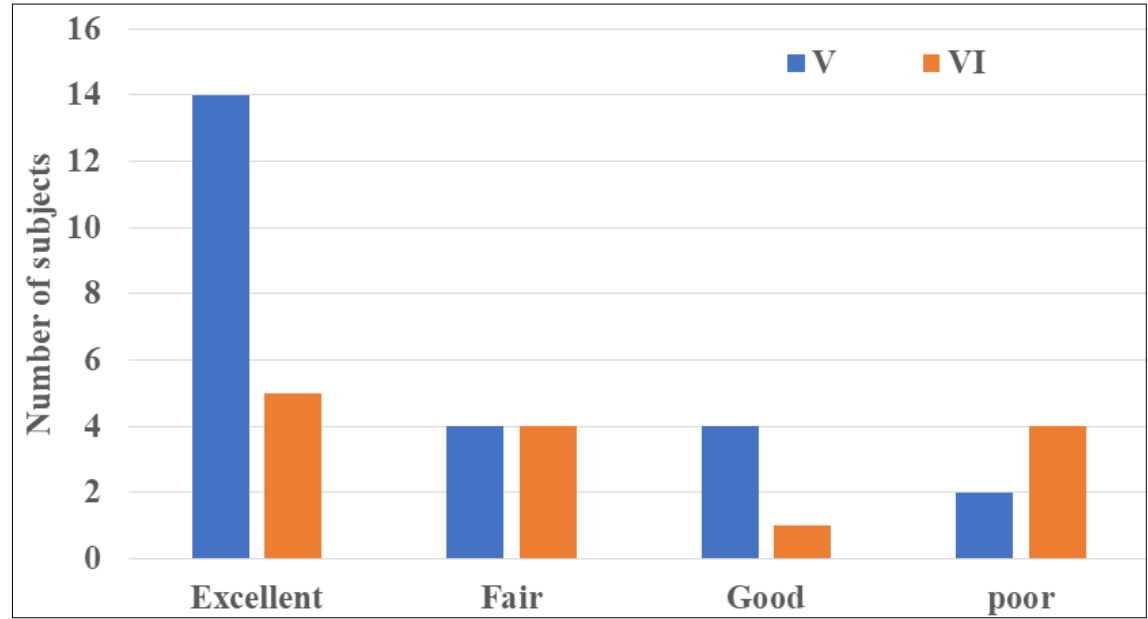

Fig 4: Distribution of subjects according to functional results and Schatzker Type

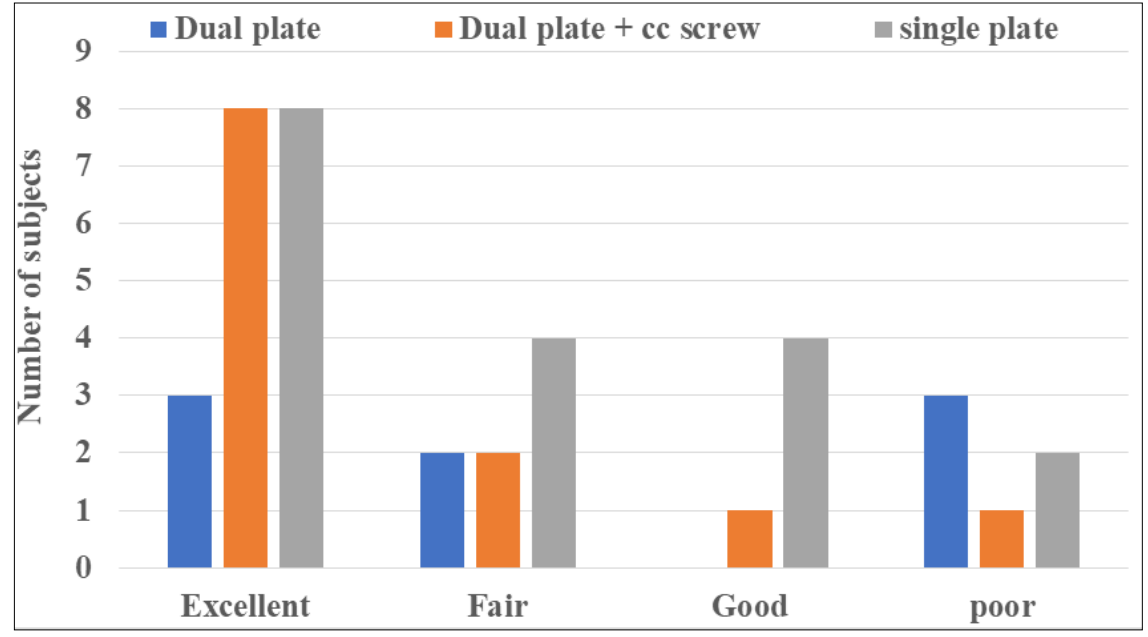

Fig 5: Distribution of subjects according to functional results and Procedure

\section{Discussion}

In modern world of development there seems to be a steep increase in complex injuries to various parts of the human body. The classification and management of tibial plateau fracture has always been a subject of debate and controversy because of their variety and complexity. In the search of perfection, any treatment modality that has a varied opinion is a subject for research and study. High energy intraarticular fractures of the tibial plateau cause ongoing problem in management and remains challenging for orthopedic surgeons [4] even to date. Schatzker type 5 and type 6 tibial plateau fractures are one among them.

In our clinical study of 45 patients, 7 patients were lost in the follow up. The analysis of the results was made in terms ofage of the patient, sex distribution, mode of injury, laterality of the fracture, analysis of the types, various open surgical modalities of treatment, complications and the subjective, clinical, radiological and functional outcome based on Honkonen and Jarvinen criteria.

In our study there were 16 females $(35.6 \%)$ and 29 males $(64.4 \%)$, ratio of male to female being $1.8: 1$. Corelates to Indian scenario where most of the females work indoors. Majority of the subjects $11(24.4 \%)$ were in the age group 3140yrs, followed by $10(22.2 \%)$ patients were in the age group $41-50 \mathrm{yrs}, 8(17.8 \%)$ patients were in the age group $>60 \mathrm{yrs}$ and $6(13.3 \%)$ patients were in the age group 21-30yrs. Minimum age was $21 \mathrm{yrs}$ and maximum age was $67 \mathrm{yrs}$.mean age of our study was $46.06+13 y$ rs.

Most number of tibial plateau fractures reported in the recent literature have resulted from high speed motor vehicle accidents and fall from height. In our study $41(91.1 \%)$ of the fractures occurred as a result of high energy road traffic accidents, 4 (8.9\%) due to fall from height. Right side \& left side involvement was $24(53.3 \%)$ and $21(46.7 \%)$ respectively. In our study, majority of the patients $36(80 \%)$ had closed fractures, 4(8.9\%) had open fracture type I and 5(11.1\%) patients had open type II fracture according to Gustilo and Anderson classification.

In the study conducted by $\mathrm{G}$ Thiruvengita Prasad et al. ${ }^{[5]}, 46$ tibial plateau fractures Schatzker type V and VI of which forty patients (33 men and 7 women) who completed the follow up. There were 20 Schatzker type V fractures and 20 Schatzker type VI fractures. In our study, 29 (64.4\%) patients had Schatzker type V fracture and $16(35.6 \%)$ patients had Schatzker type VI fracture, simulating the above study with slight type $\mathrm{V}$ preponderance.

In our study, patients were operated at an average of 1.6 days after injury ranging from 1 day to a maximum of 5 days with primary internal fixation. $27(60 \%)$ patients were operated on day $1,12(26.7 \%)$ were operated on day $2,4(8.9 \%)$ patients were operated on day $3,2(4.4 \%)$ patients were operated on day 5 in comparison with the study by Peter A cole and colleagues ${ }^{[6]}$ reported an average time for LISS implantation was 7 days after the injury, and it was performed up to 29 days after the injury, the result of our study with early operating decision were similar to the delayed procedure results.

In our study the average knee moment (flexion) was $119^{0}$, 
ranging from a minimum of 80 degrees to 140 Degrees calculated with Honkonen and Jarvinen clinical criteria was in relevance to the studies by Kataria et al. ${ }^{\text {[7] }}$ where average Knee moment $132^{\circ}$ in 48 patients for Schatzker type $5 \& 6$. marsh et al. ${ }^{[8]}$ reported that $138^{\circ}$.

The decrease in the average knee motion in our study was due to longer immobilization in postoperative period and noncompliance of patient for post-operative physiotherapy with shorter follow up.

Early results of our short follow up study are satisfactory where were comparatively similar to the experience of Schatzker et al. [9] $78 \%$ of tibial plateau fractures treated surgically had satisfactory results after 2.3 years.

Lachiewicz and Funcik. ${ }^{[10]}$ examined the operative treatment of patients with 43 tibial plateau fractures and found $81 \%$ excellent results after 2.7 years.

We achieved subjective results $31.6 \%$ had excellent, $31.6 \%$ had good, $26.3 \%$ had fair and $10.5 \%$ had poor results, most of the patients were symptom free at the end of follow up.

Clinical outcome was $42.1 \%$ had excellent, $34.2 \%$ had good, $13.2 \%$ had fair and $10.5 \%$ had poor results. Only 2 patients had extension lag of more than 10 degrees by the end of 6 months follow up. Mean flexion was 119 degrees. radiological outcome was $44.7 \%$ had excellent, $31.6 \%$ had good, $13.2 \%$ had fair and $10.5 \%$ had poor results. Articular step of more than $5 \mathrm{~mm}$ were noted in 3 patients who had poor outcomes. The same 3 patients also showed $>50 \%$ decrease in joint space compared to opposite knee and varus/valgus tilt $>10$ degree.

2 patients showed condylar widening of $>10 \mathrm{~mm}$. The above observations signifies the importance of articular reconstruction in the final outcome.

Functional results were $50 \%$ had excellent, $13.2 \%$ had good, $21.1 \%$ had fair and $15.8 \%$ had poor results. Only 2 patients in whom anatomic reduction was not achieved were unable to climb stair and squat. Walking was normal in most of them with slight limp in 6 patients. 3 patients needed the assistance of a stick. All patients returned to their preoperative employment successfully, similar in comparison to studies done by of Schatzker et al. Lachiewicz and Funcik, Hong-wei Chen et al. ${ }^{[11]}$, Tul B Pun et al. ${ }^{[12]}$.

In our study, out of 38 patients available for follow up 18 patients were single plating, 12 were single plating $+\mathrm{CC}$ screw, 8 were operated with dual plating.at the end of 6 months the functional outcomes were evaluated, there were no statistical difference in the functional outcome between the different procedures. ( $p$ value-0354) thus simulating the study by Siddaram N. Patil et al. in which 18 patients were fixed with single plate, 19 were fixed with dual plate. All patients had excellent or good HSS score ${ }^{[13]}$.

Saranjeet Singh Jagdev et al. in their study on Functional outcome of Schatzker type V and VI tibial plateau fractures managed with open reduction internal fixation using dual plates. 9 Schatzker type $\mathrm{V}$ fractures and 14 patients with Schatzker type VI fractures. The mean duration of follow-up was 68.32 months. The mean Oxford knee score for patients with Schatzker type V was 39.78. Patients with Schatzker type VI had mean OKS of 37.7.

All patients returned to their preinjury level of activity and employment ${ }^{[14]}$.

In our study, out of 38 patients available for follow up duration of 6 months of which 24 patients were Schatzker type V and 14 patients were Schatzker type VI. Among type V 14 patients had excellent result, 4 had good result, 4 had fair and 2 had poor results among type VI, 5 patients had excellent result, 4 had good result, 1 had fair and 4 had poor results. all patients returned to pre injury employment. There were no statistical difference in the functional outcome between type $\mathrm{V}$ and type VI. ( $\mathrm{p}$ value-0.221). Comparable to the above study.

There was no statistical difference in the subjective ( $\mathrm{p}$ value0.345 ) functional. ( $p$ value-0.221), clinical ( $p$ value-0.153), radiological ( $\mathrm{p}$ value-0.590) outcome between type $\mathrm{V}$ and type VI.

In our study, Majority of the patients $91.1 \%$ did not develop any complication, $4.4 \%$ of the patients developed infection, $2.2 \%$ of the patients had Impending Compartment syndrome and $2.2 \%$ of the patients had wound dehiscence. Which is significantly less compared to the study by David P Barei et al. ${ }^{[15]}$, which reported $14.5 \%$ compartment syndrome, $8.4 \%$ infections, $19.3 \%$ had deep vein thrombosis.

\section{Conclusion}

Open reduction and internal fixation by plating (dual LCP, single LCP with or without cancellous cannulated screws) of closed and open (type I and type II) Schatzker type V and VI tibial plateau fractures is an effective method of treatment. Prognosis of this complex fracture depends on

- The degree of articular reconstruction

- The integrity of the soft tissue envelope

- Post-operative physiotherapy

We found no statistical difference in assessing the Schatzker type V and VI fracture patterns. Comparable Results were achieved in radiological, clinical, subjective and functional outcomes based on Honkonen and Jarvinen criteria.

\section{Recommendations}

We recommend Open reduction and internal fixation (dual LCP, single LCP with or without cancellous cannulated screws) of closed fractures, open type I and type II Schatzker type V and VI tibial plateau fractures with moderate soft tissue injury.

We recommend for soft tissue healing before surgical intervention in case of severe soft tissue injury in view of complications like compartment syndrome, wound dehiscence, infection.

We recommend the following to take into consideration, in cases of Schatzker type V, type VI fractures.

Pre-operative analysis of fracture pattern is very important, categorization of the fracture and the pre-operative x-ray and computed tomography for planning for management. Use of fracture specific locking compression plate wherever available and early mobilization of the patient.

\section{References}

1. Hohl M. Part-I: fractures of proximal tibia and fibula. In: Rockwood C, Green D, Bucholz R, eds. Fractures in adults, $3^{\text {rd }}$ ed. Philadelphia: JB Lippincott, 1991, 17251761.

2. Honkonen SE, Jarvinen MJ. Classification of fracture of the tibial condyle. JBJS. 1992; 74B:840.

3. Koval KJ, Helfel DL. Tibial Plateau Fractures-Evaluation and treatment: J Am. Acad. Orthop. Surg. 1995; 3(2):8694.

4. Raikin S, Fromson-MI. Combined limited internal fixation with circular frame external fixation of intraarticular tibial fractures. Orthopedics. 1999; 22(11):1019.

5. Prasad GT, Kumar TS, Kumar RK, Murthy GK, 
Sundaram N. Functional outcome of Schatzker type V and VI tibial plateau fractures treated with dual plates. Indian J Orthop. 2013; 47(2):188-94.

6. Cole PA, Zlowodzki M, Kregor PJ. Treatment of proximal tibia fractures using the less invasive stabilization system: surgical experience and early clinical results in 77 fractures. J Orthop Trauma. 2004; 18(8):528-35.

7. Kataria H, Sharma N, Kanojia RK. Small wire external fixation for high-energy tibial plateau fractures. J Orthop Surg Hong Kong. 2007; 15(2):137-43.

8. Marsh JL, Smith ST, Do TT. External fixation and limited internal fixation for complex fractures of the tibial plateau. JBJS. 1995; 77(5):661.

9. Rasmussen PS. Tibial condylar fractures. Impairment of knee joint stability as an indication for surgical treatment. J Bone Joint Surg Am. 1973; 55(7):1331-50.

10. Lachiewicz PF, Funcik T. Factors influencing the results of open reduction and internal fixation of tibial plateau fractures. Clin Orthop. 1990; (259):210-5.

11. Chen HW, Chen CQ, Yi XH. Posterior tibial plateau fracture: a new treatment-oriented classification and surgical management. Int. J Clin Exp Med. 2015; 8(1):472-9.

12. Pun TB, Krishnamoorthy VP, Poonnoose PM, Oommen AT, Korula RJ. Outcome of Schatzker type V and VI tibial plateau fractures. Indian J Orthop. 2014; 48(1):3541.

13. Patil SN, Srinivas P, Bhandary D. Prospective study of management of Schatzker's type V \& VI tibial plateau fractures by different types of plate osteosynthesis. Int $\mathbf{J}$ Res Orthop. 2017; 3(5):1070-7.

14. Jagdev SS, Pathak SK, Salunke A, Maheshwari P, Ughareja P, Shah S. Functional outcome of Schatzker type $\mathrm{V}$ and VI tibial plateau fractures managed with open reduction internal fixation using dual plates. Int $\mathrm{J}$ Res Orthop. 2017; 3(5):961-5.

15. Barei PD, Nork ES, Mills JW, Henley M, Benirschke S. Complications Associated with Internal Fixation of HighEnergy Bicondylar Tibial Plateau Fractures Utilizing a Two-Incision Technique. J Orthop Trauma. 2004; 18:649-57. 